\title{
The Physics Case for an Electron-Muon Collider
}

\author{
Meng Lu, Andrew Michael Levin $\left(\mathbb{D}\right.$, Congqiao Li, Antonios Agapitos $\mathbb{D}^{\text {, }}$, Qiang Li $\mathbb{D}$, \\ Fanqiang Meng $\mathbb{D}^{D}$, Sitian Qian $\mathbb{D}$, Jie Xiao, and Tianyi Yang
}

Department of Physics and State Key Laboratory of Nuclear Physics and Technology, Peking University, Beijing 100871, China

Correspondence should be addressed to Qiang Li; qliphy0@pku.edu.cn

Received 10 December 2020; Accepted 8 February 2021; Published 26 February 2021

Academic Editor: Mariana Frank

Copyright (C) 2021 Meng Lu et al. This is an open access article distributed under the Creative Commons Attribution License, which permits unrestricted use, distribution, and reproduction in any medium, provided the original work is properly cited. The publication of this article was funded by SCOAP ${ }^{3}$.

An electron-muon collider with an asymmetric collision profile targeting multi-ab ${ }^{-1}$ integrated luminosity is proposed. This novel collider, operating at collision energies of, e.g., $20-200 \mathrm{GeV}, 50-1000 \mathrm{GeV}$, and $100-3000 \mathrm{GeV}$, would be able to probe charged lepton flavor violation and measure Higgs boson properties precisely. The collision of an electron and muon beam leads to less physics background compared with either an electron-electron or a muon-muon collider, since electron-muon interactions proceed mostly through higher-order vector boson fusion and vector boson scattering processes. The asymmetric collision profile results in collision products that are boosted towards the electron beam side, which can be exploited to reduce beam-induced background from the muon beam to a large extent. With this in mind, one can imagine a lepton collider complex, starting from colliding order $10 \mathrm{GeV}$ electron and muon beams for the first time in history and to probe charged lepton flavor violation, then to be upgraded to a collider with $50-100 \mathrm{GeV}$ electron and $1-3 \mathrm{TeV}$ muon beams to measure Higgs properties and search for new physics and finally to be transformed to a TeV-scale muon-muon collider. The cost should vary from order 100 million to a few billion dollars, corresponding to different stages, which make the funding situation more practical.

The discovery $[1,2]$ and property measurements [3-8] of the Higgs boson are a triumph of the Standard Model (SM) of particle physics and also the Large Hadron Collider (LHC). In the next 10-20 years, the LHC and the High-Luminosity LHC (HL-LHC) will be further exploring the SM and searching for physics beyond that. The 2020 update of the European Strategy for Particle Physics [9] proposes a vision for both the near- and the long-term future of the field, highlighting the need to pursue, as the highest-priority facility after the LHC, an electron-electron collider acting as a Higgs factory, such as the proposed International Linear Collider (ILC), the Compact Linear Collider (CLIC), the Future Circular Collider (FCC), or the Circular Electron Positron Collider (CEPC). These future colliders may, at various stages in their lifecycles, also serve as $\mathrm{Z}$ or top quark factories or operate at the $\mathrm{TeV}$ scale for the purpose of new physics searches. The FCC and CEPC projects also aim for an upgrade to an order $100 \mathrm{TeV}$ hadron collider. Other colliders that have been proposed with similar objectives are the High-Energy LHC (HE-
LHC) [10], a Large Hadron electron Collider (LHeC) [11], and a muon collider [12].

Recently, there has been a large amount of interest [13] in a muon-muon collider with center-of-mass energies in the multi-TeV range, which has some of the advantages of both hadron-hadron and electron-electron colliders [1416]. Because muon beams emit much less synchrotron radiation than electron beams, muons can be accelerated in a circular collider to much higher energies. On the other hand, because the proton is a composite particle, muonmuon collisions are cleaner than proton-proton collisions and also can lead to higher effective center-of-mass energies. However, due to the short lifetime of the muon, the beam-induced background (BIB) from muon decays needs to be examined and reduced properly. Based on a realistic simulation at $\sqrt{s}=1.5 \mathrm{TeV}$ with BIB included, Ref. [17] found that the coupling between the Higgs boson and the b-quark can be measured at the percent level with order $a b^{-1}$ of collected data. 
Here, we propose an electron-muon collider with an asymmetric collision profile of, e.g., $20-200 \mathrm{GeV}, 50-$ $1000 \mathrm{GeV}$, and $100-3000 \mathrm{GeV}$, for the electron-muon beam energy, respectively, corresponding to the centerof-mass energy as $126.5 \mathrm{GeV}, 447.2 \mathrm{GeV}$, and $1095.4 \mathrm{GeV}$. In history, CERN and Brookhaven scattered muons in the 1960s, using accelerator-produced muons at $\mathrm{GeV}$ scale on electrons in targets, and measured the elastic scattering cross-section [18-20]. In the late 1990s, the scattering of muons off polarized electrons was used by the SMC collaboration at CERN as a polarimeter for high-energy muon beams [21]. Recently, a new experiment, MUonE [22], has been proposed, through elastic scattering of high-energy muons at around $150 \mathrm{GeV}$ on atomic electrons, to determine the leading hadronic contribution to the muon $g$-2. A head-on electron-muon collider was first proposed in the 1990s [23-25] mainly to probe charged lepton flavor violation (CLFV) [26], which, however, has not been followed up much by the community. Here, we propose for the first time an electron-muon collider with more detailed configurations to run at both low and high energy, with broader physics goals to cover both CLFV and Higgs studies.

We now elaborate on the physics case for an electronmuon collider. The asymmetric collision profile proposed above is motivated by the fact that muons can be accelerated to higher energies much more easily than electrons. An asymmetric electron-muon collider operating at the energies proposed above can serve as an intermediate step between an electron-electron collider and a muon-muon collider. The advantages of such a novel collider are that it enables a direct study of charged lepton flavor violation in a nearly background-free environment, in contrast with other lepton colliders. The collision of an electron and a muon beam leads to less physics background compared with either an electron-electron or a muon-muon beam because the background physics processes are mostly higher-order vector boson fusion or scattering processes. Moreover, the asymmetric beam profile tends to create collision products that are boosted towards the electron beam side, which can be exploited to reduce muon BIB from muon beam upstream to a large extent, for example, by adding a shielding nozzle in the muon side with a large cone size without much loss on acceptance.

The cross-section dependence on the center-of-mass energy for six of the dominant SM processes at an electronmuon collider is shown in Figure 1. The two leading processes in Figure 1 are the vector boson fusion or scattering processes $e^{-} \mu^{+} \longrightarrow v_{e} \tilde{v}_{\mu} \mathrm{Z}$ and $e^{-} \mu^{+} \longrightarrow v_{e} \tilde{v}_{\mu} \mathrm{H}$. The crosssection of $e^{-} \mu^{+} \longrightarrow v_{e} \tilde{v}_{\mu} \mathrm{Z}$ is $291 \mathrm{fb}(863 \mathrm{fb})$ at $\sqrt{s}=0.5 \mathrm{TeV}$ $(1.0 \mathrm{TeV})$, and the cross-section of $e^{-} \mu^{+} \longrightarrow v_{e} \tilde{v}_{\mu} \mathrm{H}$ is $75 \mathrm{fb}$ $(209 \mathrm{fb})$ at $\sqrt{s}=0.5 \mathrm{TeV}(1.0 \mathrm{TeV})$.

An electron-muon collider could operate at multiple energies to search for CLFV in $e \mu \longrightarrow \mathrm{Z}$ and $\mathrm{H}$, or $e \mu \longrightarrow$ $e e$, etc. Here, we take Higgs CLFV as an example (some other examples are discussed in [26]), which is interesting because its observation may provide insight into some fundamental questions in nature, e.g., whether there is a secondary mech- anism for the electroweak symmetry breaking. $\mathrm{H} \longrightarrow e \mu$ has already been studied at the LHC $[27,28]$, and the most stringent limit so far, from ATLAS, is $\mathrm{Br}(\mathrm{H} \longrightarrow e \mu)<6.2 \times 10^{-5}$ [28]. The CEPC projected limit is $\mathrm{Br}(\mathrm{H} \longrightarrow e \mu)<1.2 \times 10^{-5}$ [29]. In this paper, we implement the Higgs CLFV model [27] in MadGraph5_aMC@NLO [30], with the CLFV Yukawa couplings $\left|Y_{e \mu}\right|$ set by default to match the current limit on $\mathrm{Br}(\mathrm{H} \longrightarrow e \mu)$ from Ref. [28]. In Figure 2, we show an energy scan near $\sqrt{s}=125 \mathrm{GeV}$ (with, e.g., $20-200 \mathrm{GeV}$ for the electron-muon beam energy) for Higgs CLFV production $e \mu \longrightarrow \mathrm{H} \longrightarrow \mathrm{b} \overline{\mathrm{b}}$ with and without initial state radiation (ISR) effects [31, 32], and the dominant background $e \mu \longrightarrow$ $v_{e} \tilde{v}_{\mu} \mathrm{Z} \longrightarrow v_{e} \tilde{v}_{\mu} \mathrm{b} \overline{\mathrm{b}}$ at an electron-muon collider. The signal peak cross-section is around $5.3 \mathrm{pb}$, while the background cross-section is around $0.1 \mathrm{fb}$. Based on a simple estimation with the signal and background yields, with $1 \mathrm{fb}^{-1}$ of data at around $\sqrt{s}=125 \mathrm{GeV}$, an electron-muon collider should already be able to obtain a 100 times better limit on Higgs CLFV than the current best result from ATLAS. As a caveat, the actual Higgs production rate will depend on the convolution of the muon beam energy profile with the Higgs production cross-section, not just the peak cross-section, as discussed in Ref. [14].

An electron-muon collider can also serve as a Higgs factory, with asymmetric collision profile of, e.g., $50-1000 \mathrm{GeV}$ and $100-3000 \mathrm{GeV}$ for the electron-muon beam energy, respectively, corresponding to the center-of-mass energy as $447.2 \mathrm{GeV}$ and $1095.4 \mathrm{GeV}$. We would like to highlight two advantages of an electron-muon collider: (1) as there is no CLFV in the SM, one gets less physics backgrounds compared with the case of other same-flavor leptonic colliders. The dominant background to $e^{-} \mu^{+} \longrightarrow v_{e} \tilde{v}_{\mu} \mathrm{H}$ with $\mathrm{H} \longrightarrow$ $\mathrm{b} \overline{\mathrm{b}}$ is $e^{-} \mu^{+} \longrightarrow v_{e} \tilde{v}_{\mu} \mathrm{Z}$ with $\mathrm{Z} \longrightarrow \mathrm{b} \overline{\mathrm{b}}$ which can be further reduced effectively by imposing selections on the invariant mass of di-b jets; (2) the asymmetric beam energies tend to have collision products boosted towards the electron beam side, as shown in Figure 3, which can be exploited to reduce muon BIB to a large extent, for example, by adding a shielding nozzle in the muon side with large cone size without much loss on acceptance.

The cross-section of $e^{-} \mu^{+} \longrightarrow v_{e} \tilde{v}_{\mu} \mathrm{H}$ with $\mathrm{H} \longrightarrow \mathrm{b} \overline{\mathrm{b}}$ depends on

$$
\sigma=\sigma(v v \mathrm{H}) \cdot \mathrm{BR}(\mathrm{H} \longrightarrow \mathrm{b} \overline{\mathrm{b}})=\frac{g_{\mathrm{HWW}}^{2} g_{\mathrm{Hbb}}^{2}}{\Gamma_{\mathrm{H}}}
$$

where $g_{\mathrm{HWW}}$ is the coupling of the Higgs boson to the W boson, $g_{\mathrm{Hbb}}$ is the coupling of the Higgs boson to the b quark, and $\Gamma_{\mathrm{H}}$ is the Higgs boson width. From the formula above, we can further obtain the uncertainty of $g_{\mathrm{Hbb}}$ as

$$
\frac{\Delta g_{\mathrm{Hbb}}}{g_{\mathrm{Hbb}}}=\frac{1}{2} \sqrt{\left(\frac{\Delta \sigma}{\sigma}\right)^{2}+\left(\frac{\Delta g_{\mathrm{HWW}}^{2} / \Gamma_{\mathrm{H}}}{g_{\mathrm{HWW}}^{2} / \Gamma_{\mathrm{H}}}\right)^{2}} .
$$

To roughly estimate the precision of a $g_{\mathrm{Hbb}}$ measurement with an electron-muon collider, we assume that $g_{\mathrm{HWw}}^{2} / \Gamma_{\mathrm{H}}$ 


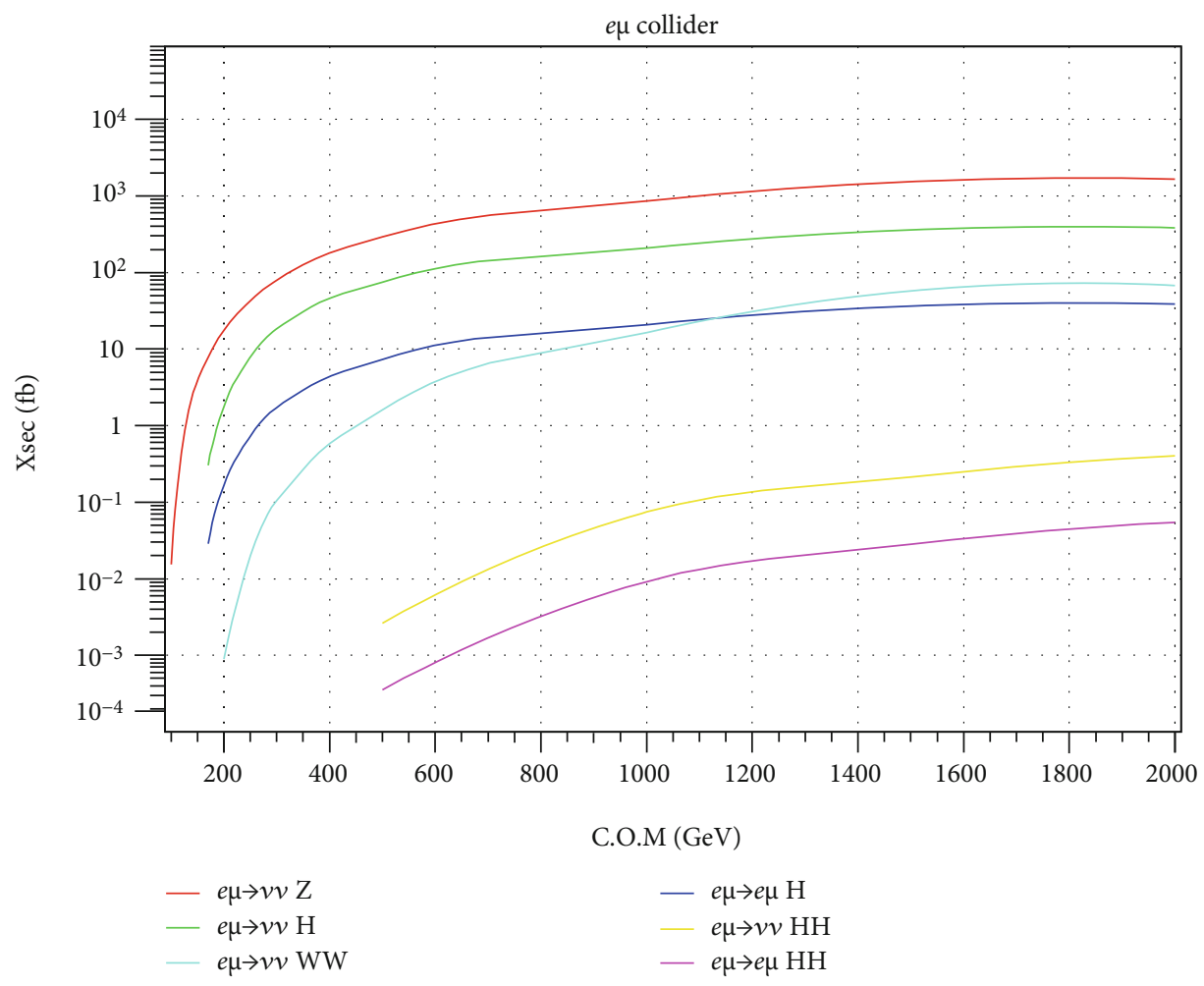

FIGURE 1: Cross-section dependence on center-of-mass energy of six dominant physics processes at an electron-muon collider.

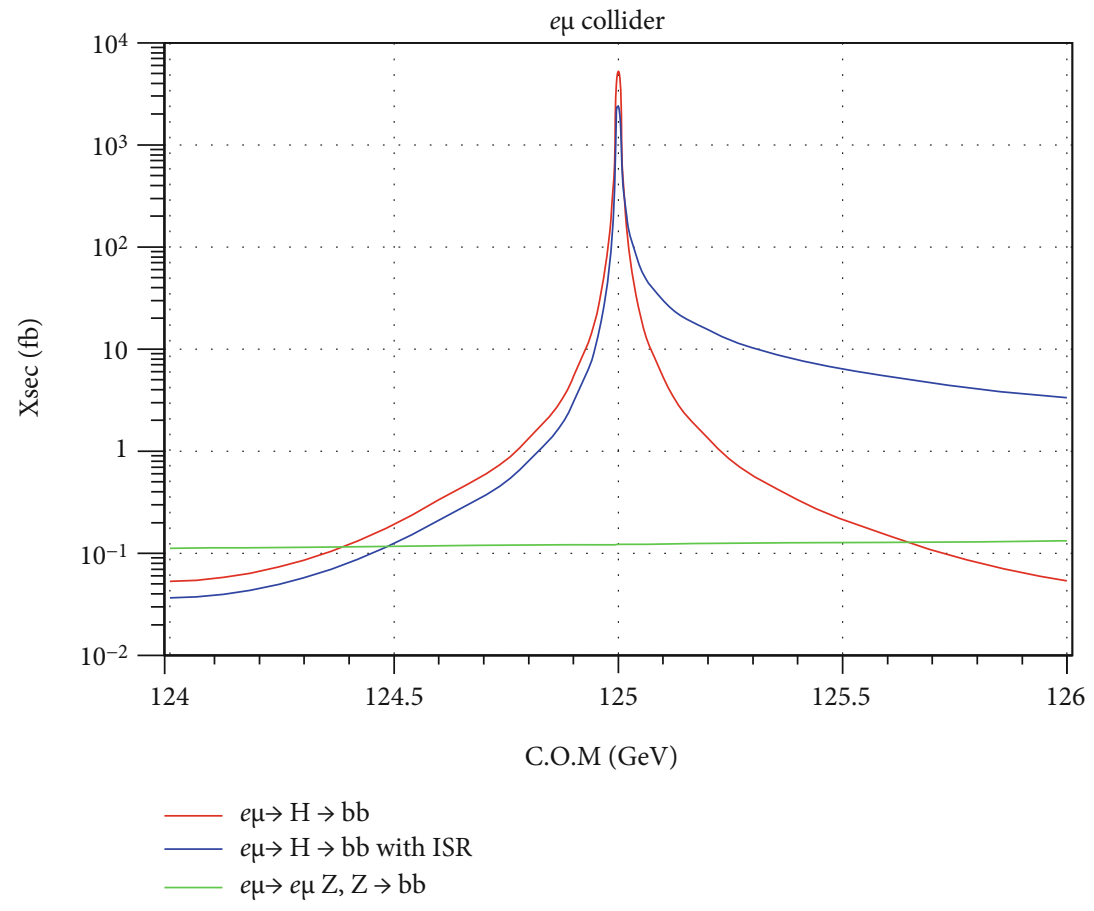

Figure 2: Energy scan near $\sqrt{s}=125 \mathrm{GeV}$ for Higgs CLFV production $e \mu \longrightarrow \mathrm{H} \longrightarrow \mathrm{b} \overline{\mathrm{b}}$ and the dominant background $e \mu \longrightarrow \nu v \mathrm{Z} \longrightarrow \nu v \mathrm{~b} \overline{\mathrm{b}}$ at an electron-muon collider. The CLFV Yukawa couplings $\left|Y_{e \mu}\right|$ is set by default to match the current limit on $\operatorname{Br}(\mathrm{H} \longrightarrow e \mu)$ from ATLAS experiment [28]. 


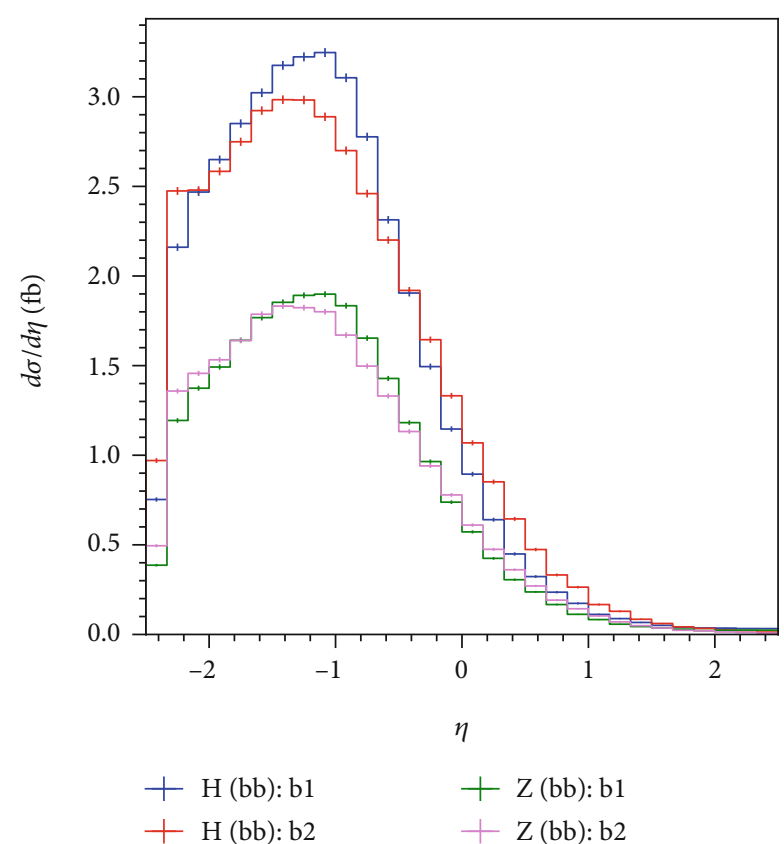

Figure 3: The $\eta$ distributions of the leading and subleading $b$-jet from Higgs and $\mathrm{Z}$ boson decay. The electron-beam $(100 \mathrm{GeV})$ and muon-beam $(3000 \mathrm{GeV})$ come from the negative and positive $\eta$ partition. The detector simulation is implemented through Delphes using the muon detector configuration. The Valencia algorithm [37] is used for jet reconstruction with the jet cone size as 0.5 . The b-jets are required with $p_{\mathrm{T}}>20 \mathrm{GeV}$ and $|\eta|<2.5$, with the b-tagging efficiency $70 \%$. The $\eta$ distributions indicate that jets are boosted towards the electron-beam side.

can be measured with the same precision as in CLIC [33], with a $3 \%$ uncertainty conservatively. The cross-section uncertainty can be approximated as

$$
\frac{\Delta \sigma}{\sigma} \simeq \frac{\sqrt{N+B}}{N}
$$

where $N$ and $B$ are the number of $\mathrm{H} \longrightarrow \mathrm{b} \overline{\mathrm{b}}$ and background events, respectively.

$\mathrm{H} \longrightarrow \mathrm{b} \overline{\mathrm{b}}$ and $\mathrm{Z} \longrightarrow \mathrm{b} \overline{\mathrm{b}}$ samples are generated using MadGraph5_aMC@NLO [30] and interfaced with Pythia [34] for parton showering and hadronization. A fast simulation is performed using Delphes version 3 [35] with the muon collider configuration. We require that the leading and subleading b-tagged jets satisfy $p_{\mathrm{T}}>40 \mathrm{GeV}$ and -2.5 $<\eta<1$ (corresponding to a $40.4^{\circ}$ shielding nozzle on the muon beam side). Figure 4 shows the invariant mass distribution of the two b-tagged jets after this selection.

We require that $100<m_{\mathrm{bb}}<150 \mathrm{GeV}$ to suppress the background and then evaluate the uncertainty on the crosssection based on equation (3). Taking into account the $g_{\mathrm{HWW}}^{2} / \Gamma_{\mathrm{H}}$ uncertainty, we estimate the precision of $g_{\mathrm{Hbb}}$ in different collision schemes using the number of the signal and background events after the selection. The results are listed in Table 1, showing that the measured precision of $g_{\mathrm{Hbb}}$ at an electron-muon collider is at the few percent level

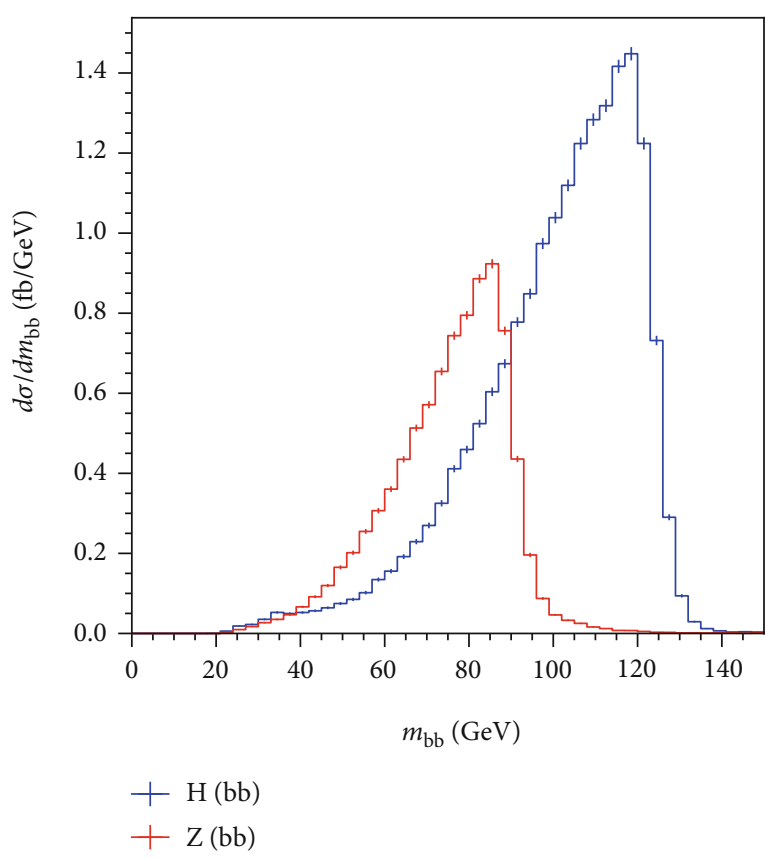

Figure 4: The invariant mass distribution of two b-tagged jets for the signal $\mathrm{H} \longrightarrow \mathrm{b} \bar{b}$ and background $\mathrm{Z} \longrightarrow \mathrm{b} \bar{b}$ process, after requiring $p_{\mathrm{T}}^{\mathrm{b}}>40 \mathrm{GeV}$ and $-2.5<\eta^{\mathrm{b}}<1$, at a $100-3000 \mathrm{GeV}$ electron-muon collider.

TABLE 1: Summary of collider parameters and the corresponding uncertainties on the Higgs boson coupling to $b$-quarks. $\sqrt{s}=447.2$ (1095.3) GeV corresponds to a 50 (100) GeV electron beam and a 1 (3) $\mathrm{TeV}$ muon beam.

\begin{tabular}{lcccc}
\hline$L_{\mathrm{int}}\left(\mathrm{ab}^{-1}\right)$ & $\sqrt{s}(\mathrm{GeV})$ & $\frac{\Delta \sigma}{\sigma}(\%)$ & $\frac{\Delta g_{\mathrm{HWw}}^{2} / \Gamma_{\mathrm{H}}}{g_{\mathrm{HWw}}^{2} / \Gamma_{\mathrm{H}}}(\%)$ & $\left(\Delta g_{\mathrm{Hbb}} / g_{\mathrm{Hbb}}\right)(\%)$ \\
\hline \multirow{2}{*}{0.5} & 447.2 & 2.5 & 3 & 2.0 \\
& 1095.4 & 1.4 & 3 & 1.7 \\
\multirow{2}{*}{1.5} & 447.2 & 1.4 & 3 & 1.7 \\
& 1095.4 & 0.8 & & 1.6 \\
2.0 & 447.2 & 1.2 & 3 & 1.6 \\
& 1095.4 & 0.7 & 3 & 1.6 \\
\hline
\end{tabular}

with order $\mathrm{ab}^{-1}$ of data and is dominated by the uncertainty on $g_{\mathrm{HWw}}^{2} / \Gamma_{\mathrm{H}}$.

The physical infrastructure needed to generate electron and muon beams with energies below $100 \mathrm{GeV}$ would be either a small linear or circular accelerator. A muon beam at the $\mathrm{TeV}$ scale can be achieved in a kilometer-sized storage ring. A set-up that can generate both electron and positron beams, or both muon and antimuon beams, or polarized beams, should be considered to further enrich the physics outcome. Such a lepton collider complex can start from colliding order $10 \mathrm{GeV}$ electron and muon beams for the first time in history and to probe CLFV, then to be upgraded to a collider with $50-100 \mathrm{GeV}$ electron and $1-3 \mathrm{TeV}$ muon beams to measure Higgs properties and probe new physics beyond the SM including CLFV further and finally to be transformed to a $\mathrm{TeV}$-scale muon-muon collider. The cost should vary from order 100 million to a few billion dollars 
[36], corresponding to different stages, which make the funding situation more practical. Note that such a lepton collider complex can also serve as a muon source at various energy scales for general science research.

In summary, we have proposed a novel collider, with colliding electron and muon beams with asymmetric energies, and shown that it has great potential to probe charged lepton flavor violation and to measure Higgs properties precisely. An electron-muon collider has less physics backgrounds than either an electron-electron collider or a muon-muon collider. The asymmetric beam energies lead to collision products that are boosted towards the electron beam side, which can be exploited to reduce muon BIB to a large extent. Such a collider can operate at center-of-mass energies up to a few $\mathrm{TeV}$ and can serve as an intermediate step towards a future higher-energy physics facility. We urge the community to consider this new option seriously.

\section{Data Availability}

The data used to support the findings of this study are included within the article.

\section{Disclosure}

The work has appeared as a preprint on arXiv: https://arxiv .org/abs/2010.15144.

\section{Conflicts of Interest}

The authors declare that they have no conflicts of interest.

\section{Acknowledgments}

This work is supported in part by the National Natural Science Foundation of China under Grant No. 12075004 and by MOST under grant No. 2018YFA0403900.

\section{References}

[1] CMS Collaboration, "Observation of a new boson at a mass of $125 \mathrm{GeV}$ with the CMS experiment at the LHC," Physics Letters B, vol. 716, p. 30, 2012.

[2] ATLAS Collaboration, "Observation of a new particle in the search for the Standard Model Higgs boson with the ATLAS detector at the LHC," Physics Letters B, vol. 716, no. 1, pp. 129, 2012.

[3] The CMS Collaboration, "Observation of a new boson with mass near $125 \mathrm{GeV}$ in pp collisions at $\sqrt{ } \mathrm{s}=7$ and $8 \mathrm{TeV}$," Journal of High Energy Physics, vol. 2013, no. 6, p. 81, 2013.

[4] The ATLAS Collaboration, "Measurements of Higgs boson production and couplings in diboson final states with the ATLAS detector at the LHC," Physics Letters B, vol. 726, no. 1-3, pp. 88-119, 2013.

[5] The ATLAS Collaboration, "Evidence for the spin-0 nature of the Higgs boson using ATLAS data," Physics Letters B, vol. 726, pp. 120-144, 2013.

[6] The CMS Collaboration, "Precise determination of the mass of the Higgs boson and tests of compatibility of its couplings with the standard model predictions using proton collisions at 7 and $8 \mathrm{TeV}$," The European Physical Journal C, vol. 75, p. 212, 2015.

[7] The CMS Collaboration, "Constraints on the spin-parity and anomalous HVV couplings of the Higgs boson in proton collisions at 7 and 8 TeV," Physical Review D, vol. 92, article 012004, 2015.

[8] The ATLAS Collaboration and CMS Collaboration, "Combined measurement of the Higgs boson mass in pp collisions at $\sqrt{ } \mathrm{s}=7$ and $8 \mathrm{TeV}$ with the ATLAS and CMS experiments," Physical Review Letters, vol. 114, article 191803, 2015.

[9] https://europeanstrategy.cern/.

[10] X. C. Vidal, M. d'Onofrio, P. J. Fox et al., "Report from Working Group 3 : Beyond the Standard Model physics at the HL-LHC and HE-LHC," CERN Yellow Reports: Monographs, vol. 7, pp. 585-865, 2019.

[11] J. L. Abelleira Fernandez, C. Adolphsen, A. N. Akay et al., "A Large Hadron Electron Collider at CERN Report on the Physics and Design Concepts for Machine and Detector," Journal of Physics G: Nuclear and Particle Physics, vol. 39, no. 7, article 075001, 2012.

[12] J. P. Delahaye, M. Diemoz, K. Long et al., "Muon colliders," 2019, http://arxiv.org/abs/1901.06150.

[13] D. Schulte, N. Pastrone, and K. Long, "Sketching out a muon collider," CERN Courier, vol. 60, no. 3, pp. 41-46, 2020.

[14] M. Greco, T. Han, and Z. Liu, "ISR effects for resonant Higgs production at future lepton colliders," Physics Letters B, vol. 763, pp. 409-415, 2016.

[15] A. Costantini, F. De Lillo, F. Maltoni et al., "Vector boson fusion at multi-TeV muon colliders," Journal of High Energy Physics, vol. 2020, p. 80, 2020.

[16] D. Buttazzo, D. Redigolo, F. Sala, and A. Tesi, "Fusing vectors into scalars at high energy lepton colliders," Journal of High Energy Physics, vol. 2018, no. 11, p. 144, 2018.

[17] N. Bartosik, A. Bertolin, L. Buonincontri et al., "Detector and physics performance at a muon collider," Journal of Instrumentation, vol. 15, no. 5, article P05001, 2020.

[18] G. Backenstoss, B. D. Hyams, G. Knop, P. C. Marin, and U. Stierlin, "Scattering of $8-\mathrm{GeV} \mu$ Mesons on electrons," Physics Review, vol. 129, no. 6, pp. 2759-2765, 1963.

[19] T. Kirk and S. Neddermeyer, "Scattering of high-energy positive and negative muons on electrons," Physical Review, vol. 171, article 1412, 1968.

[20] P. L. Jain and N. J. Wixon, "Scattering of high-energy positive and negative muons on electrons," Physical Review Letters, vol. 23, p. 715, 1969.

[21] D. Adams, B. Adeva, T. Akdogan et al., "Measurement of the SMC muon beam polarisation using the asymmetry in the elastic scattering off polarised electrons," Nuclear Instruments and Methods in Physics Research Section A: Accelerators, Spectrometers, Detectors and Associated Equipment, vol. 443, no. 1, pp. 1-19, 2000.

[22] G. Abbiendi, C. M. C. Calame, U. Marconi et al., "Measuring the leading hadronic contribution to the muon g-2 via $\mu$ e scattering," European Physical Journal C: Particles and Fields, vol. 77, no. 3, p. 139, 2017.

[23] G. W.-S. Hou, "Possible resonances in $\mu+\mathrm{e}-\rightarrow \mu-\mathrm{e}+$ collisions," Nuclear Physics B - Proceedings Supplements, vol. 51, no. 1, pp. 40-49, 1996.

[24] S. Y. Choi, C. S. Kim, Y. J. Kwon, and S. H. Lee, "High energy FCNC search throughe $\mu$ colliders," Physical Review D, vol. 57, no. 11, pp. 7023-7026, 1998. 
[25] V. Barger, S. Pakvasa, and X. Tata, “Are e $\mu$ colliders interesting?”, Physics Letters B, vol. 415, no. 2, pp. 200-204, 1997.

[26] F. Bossi and P. Ciafaloni, "Lepton flavor violation at muonelectron colliders," Journal of High Energy Physics, vol. 2020, no. 10, p. 33, 2020.

[27] The CMS Collaboration, "Search for lepton flavour violating decays of the Higgs boson to e $\tau$ and $\mathrm{e} \mu$ in proton-proton collisions at $\sqrt{ } s=8 \mathrm{TeV}$," Physics Letters $B$, vol. 763, pp. 472500, 2016.

[28] The ATLAS Collaboration, "Search for the Higgs boson decays $\mathrm{H} \rightarrow$ ee and $\mathrm{H} \rightarrow \mathrm{e} \mu$ in pp collisions at $\sqrt{ } \mathrm{s}=13 \mathrm{TeV}$ with the ATLAS detector," Physics Letters B, vol. 801, article 135148, 2020.

[29] Q. Qin, Q. Li, C. D. Lü, F. S. Yu, and S. H. Zhou, "Charged lepton flavor violating Higgs decays at future e + e - colliders," The European Physical Journal C, vol. 78, p. 835, 2018.

[30] J. Alwall, R. Frederix, S. Frixione et al., "The automated computation of tree-level and next-to-leading order differential cross sections, and their matching to parton shower simulations," Journal of High Energy Physics, vol. 1407, p. 79, 2014.

[31] Q. Li and Q.-S. Yan, "Initial state radiation simulation with MadGraph,” http://arxiv.org/abs/1804.00125.

[32] C. Z. Cheng Chen, G. Li, Q. Li, M. Ruan, L. Wang, and Q. S. Yan, " $\mathrm{H} \rightarrow \mathrm{e}+\mathrm{e}-$ at the CEPC: initial state radiation effect with MadGraph," Journal of Physics G: Nuclear and Particle Physics, vol. 45 , no. 1 , article $015004,2018$.

[33] H. Abramowicz, A. Abusleme, K. Afanaciev et al., "Higgs physics at the CLIC electron-positron linear collider," European Physical Journal C: Particles and Fields, vol. 77, no. 7, p. 475, 2017.

[34] T. Sjostrand, S. Ask, J. R. Christiansen et al., "An introduction to PYTHIA 8.2," Computer Physics Communications, vol. 191, pp. 159-177, 2015.

[35] DELPHES 3 Collaboration, "DELPHES 3: a modular framework for fast simulation of a generic collider experiment," Journal of High Energy Physics, vol. 1402, p. 57, 2014.

[36] V. Shiltsev, "Considerations on energy frontier colliders after LHC," 2017, http://arxiv.org/abs/1705.02011.

[37] M. Boronat, J. Fuster, I. García, E. Ros, and M. Vos, “A robust jet reconstruction algorithm for high-energy lepton colliders," Physics Letters B, vol. 750, pp. 95-99, 2015. 